\title{
Diffuse cutaneous involvement and sinus histiocytosis with massive lymphadenopathy
}

\author{
A. A. WoODCOCK \\ B.Sc., M.B., Ch.B., M.R.C.P. \\ P. L. DE V. Hart \\ M.B.(Lond.), F.R.C.P.
}

General Hospital, Bandar Seri Begawan, Brunei

\begin{abstract}
Summary
Severe skin involvement complicated a case of sinus histiocytosis with massive lymphadenopathy in a young Malay girl. The clinical and histological features of this rare manifestation are described.

\section{Introduction}

Sinus histiocytosis with massive lymphadenopathy (SHML) is a recently described benign illness of unknown aetiology with a protracted course unmodified by any known therapy. It is characterized by cervical lymphadenopathy (often bilateral and massive), neutrophil leucocytosis and polyclonal hypergammaglobulinaemia. Involvement of other lymph node groups is common, but extra-nodal
\end{abstract}

manifestations are uncommon. Initial descriptions of the disease (Rosai and Dorfman, 1969) specifically excluded cutaneous manifestations but more recently, 7 cases have been described with multiple skin nodules (Thawerani et al., 1978). This report describes a typical case of SHML in a young Malay girl with the subsequent development of severe skin involvement manifesting itself as a rash resembling psoriasis.

\section{Case report}

A 12-year-old Malay girl presented in January 1976 with a 6-month history of painless swelling of the neck due to massive discrete cervical lymphadenopathy (Fig. 1). Inguinal and axillary nodes

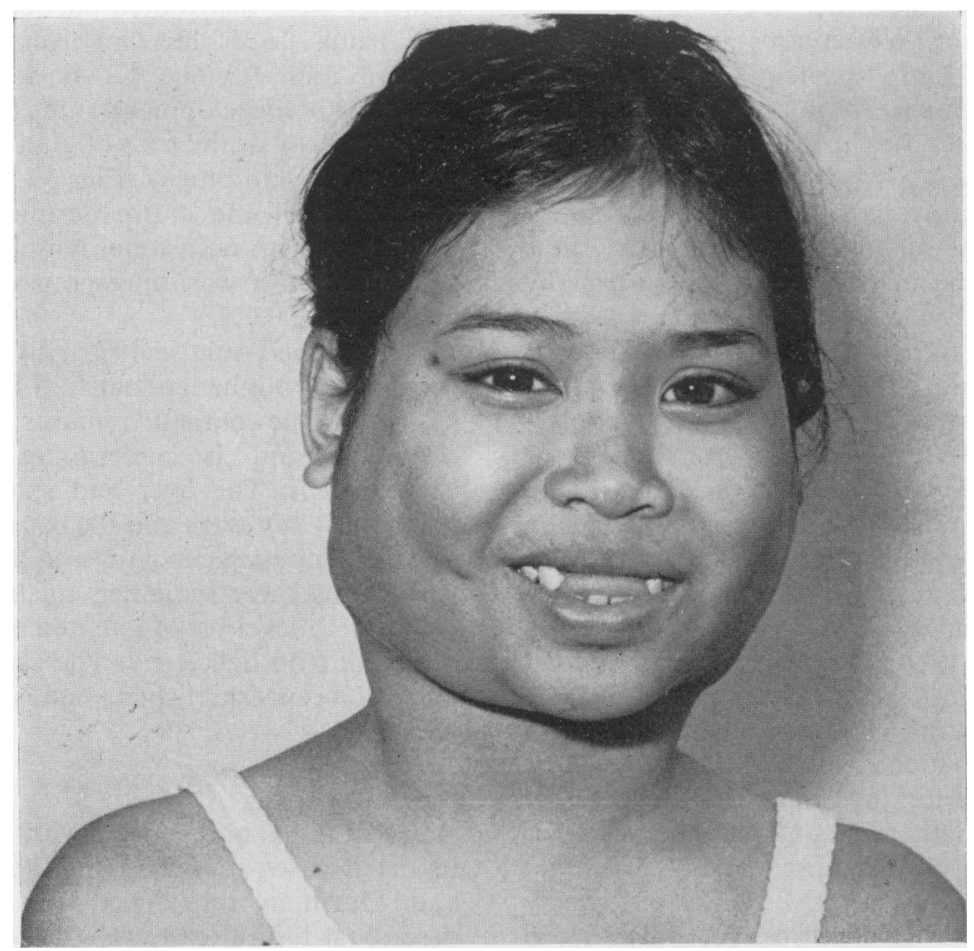

FIG. 1. Gross cervical lymphadenopathy July 1976. 


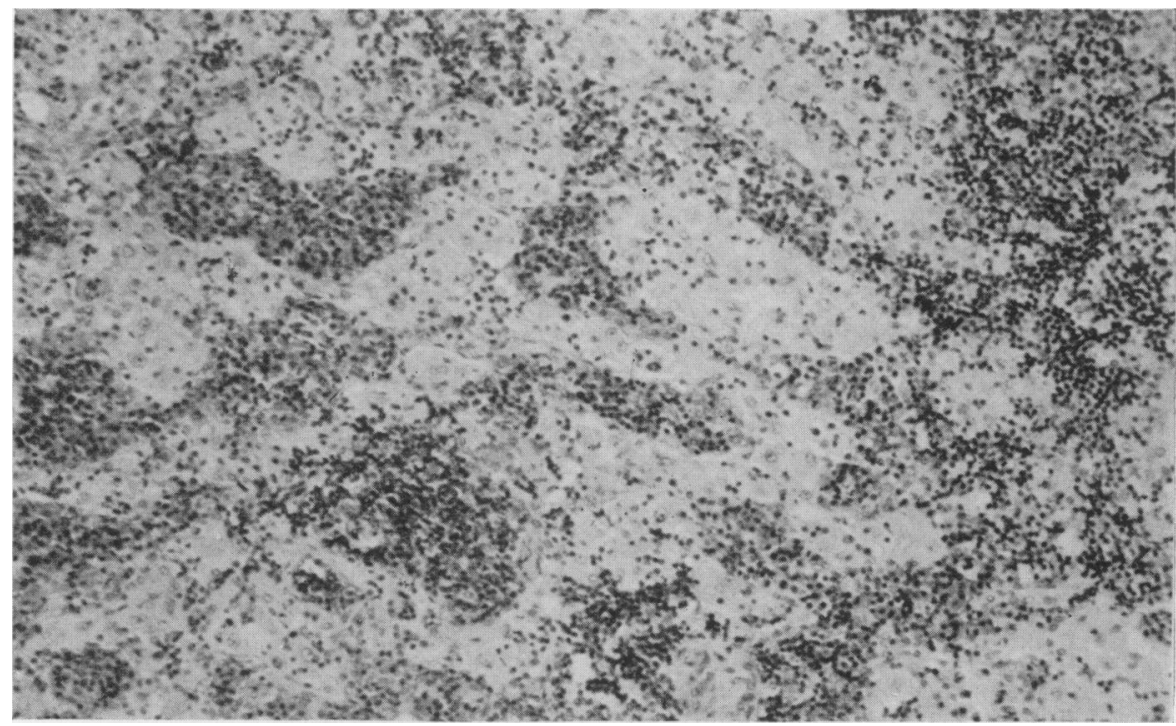

FIG. 2. Lymph node biopsy showing prominent sinus histiocytosis, infrequent lymphoid follicles and numerous plasma cells in the intersinusoidal cords $(\mathrm{HE}, \times 132)$.

were also enlarged to a lesser degree. There was neither fever nor hepatosplenomegaly and her general physical condition was excellent. Investigations showed: $\mathrm{Hb} 8.4 \mathrm{~g} / \mathrm{dl}$; WCC $21.9 \times 10^{\circ} / \mathrm{l}$; ESR $147 \mathrm{~mm}$ in 1st hr (Westergren); serum albumin $29 \mathrm{~g} / \mathrm{l}$; globulin $73 \mathrm{~g} / \mathrm{l}$; protein electrophoresis showed a polyclonal rise in gamma globulin; IgG $40 \mathrm{~g} / 1$ (normal 6-16 g/l); IgA $5.6 \mathrm{~g} / 1$ (1-4); IgM $2.7 \mathrm{~g} / 1(0 \cdot 5-1 \cdot 2)$. Chest X-ray showed gross bilateral hilar lymphadenopathy. A cervical gland biopsy showed the typical histology of SHML; sinus histiocytosis with plasma cell infiltrates especially in the intersinoidal cords and infrequent widely separated lymphoid follicles (Fig. 2). Stains for acidfast bacilli, fungi and protozoa were negative. The following serological tests were negative: toxoplasmosis (1 in 40); EB virus; Candida sp.; Mucor sp.; Histoplasma; Cryptococcus neoformans. Whilst in hospital, the patient developed an episode of chicken pox which progressed in the usual manner with clinical recovery and resolution of the skin lesions.

A therapeutic trial of doxycycline produced no response. By May 1976, the Toxoplasma haemagglutination inhibition test (HAI) had become strongly positive (titre 1/640), a significant rise from January 1976. The patient owned 11 cats. However, the Toxoplasma dye test was negative and a therapeutic trial of pyrimethamine and sulphadiazine for 6 weeks, produced no response. The Toxoplasma dye test has remained negative, despite strongly positive Toxoplasma HAI titres (between 1/640 and $1 / 2560$ ) until the present time.
In August 1977, there was gradual onset of a non-ce itchy rash resembling guttate psoriasis. Discrefe red scaling papules ranging from 0.3 to $1.5 \mathrm{~cm}$ in diameter were distributed particularly over the faces and trunk, with less involvement of the limbs $\overrightarrow{5}$ (Figs 3 and 4). The Koebner phenomenon wase present, with development of the rash along scratches and especially at the sites of gland biopsy 18 months $\stackrel{\circ}{\rightarrow}$ previously. Skin biopsy (Figs 5 and 6) showed dense윽 infiltration of the entire dermis by aggregates of histiocytes with occasional lymphocytes and plasma cells. The rash was unresponsive to topical betamethasone $0.05 \%$.

At present (August 1979), in spite of the gross: persisting lymphadenopathy (4 years) and rash 3 . ( 2 years) the patient remains in good physicalo condition and is undergoing normal pubertal 3 development. The liver and spleen have not been ${ }_{0}$ palpable at any stage and she has remained apyrexial. The neutrophil leucocytosis and polyclonal rise ino $\gamma$-globulins have remained unchanged throughout, but the $\mathrm{Hb}$ level has fluctuated as a consequence of $\sigma$ recurrent iron deficiency. The cause of the iron loss N has not been established, but is perhaps unrelated N to SHML.

\section{Discussion}

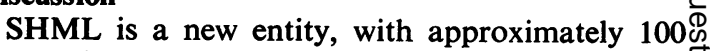
reported cases in the world literature since Rosai ${ }_{0}$ and Dorfman introduced the term in 1969 to describe 4 typical cases. Extra-nodal manifestations $\overparen{\odot}$ of SHML are being reported more frequently than $\frac{}{\circ}$ 


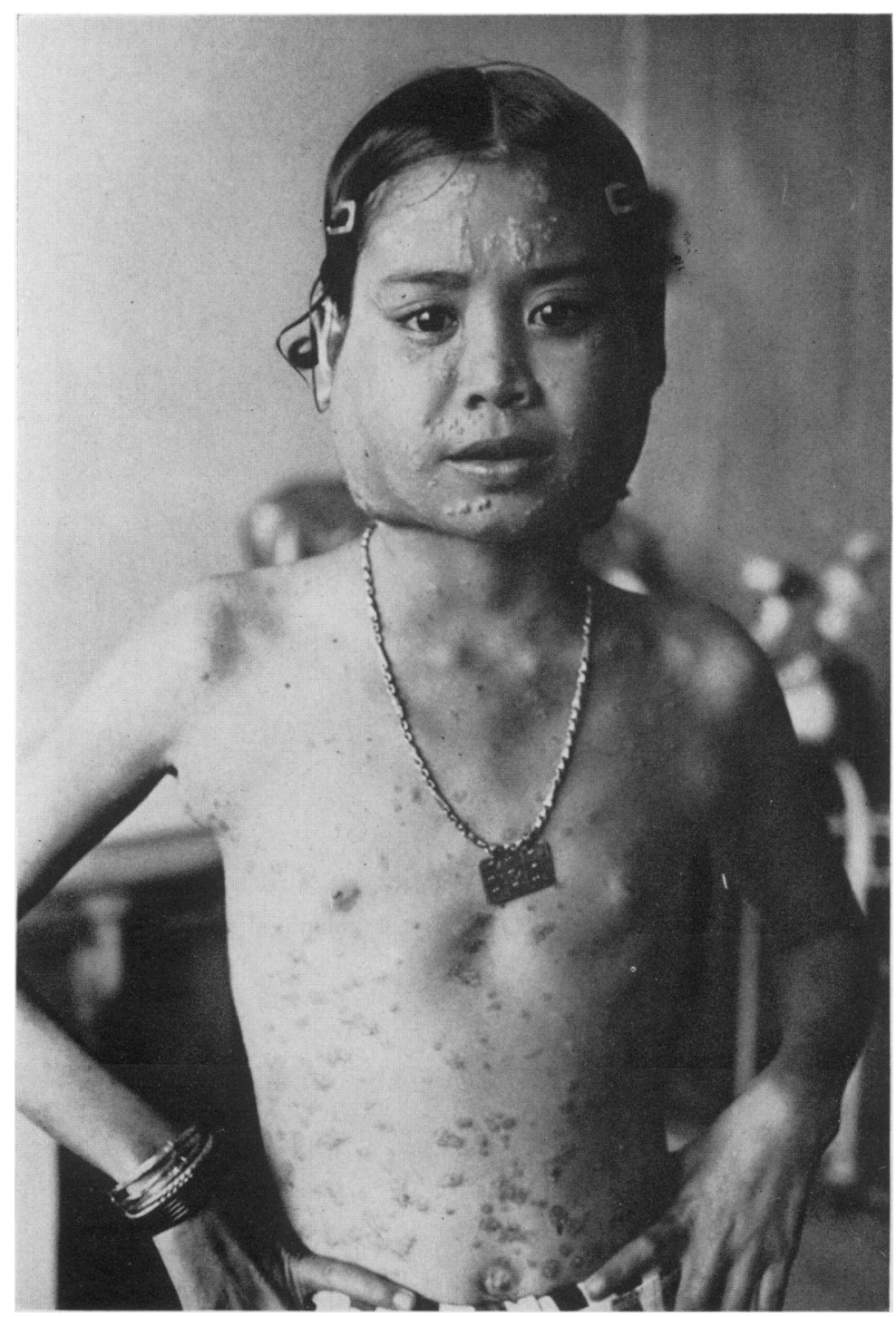

Fig. 3. Discrete red scaling papules over face and trunk.

indicated in the original descriptions, but are still uncommon. A review of the world literature on cutaneous manifestations of SHML discovered 7 cases of multiple skin lesions (Thawerani et al., 1978). They were described as nodular, often xanthomatous, in appearance.

At presentation, this patient had the characteristic histological and clinical picture of SHML. Cutaneous lesions resembling guttate psoriasis developed 18 months after the onset of lymphadenopathy. The cutaneous lesions are macroscopically unlike those previously described in this condition, but the histological picture of dermal infiltration with aggregates of histocytes, plasma cells and lymphocytes is similar to the findings in other cases of cutaneous involvement in SHML and excludes psoriasis. The exact aetiology of the disease is uncertain, despite widespread searches for an infectious agent or immune defect. The significance of elevated antibodies to Klebsiella sp. (Lampert and Lennert, 1976) is not certain. The raised Toxoplasma HAI titres in this patient is probably a non-specific finding secondary to hypergammaglobulinaemia. Toxoplasma HAI titres have not been 


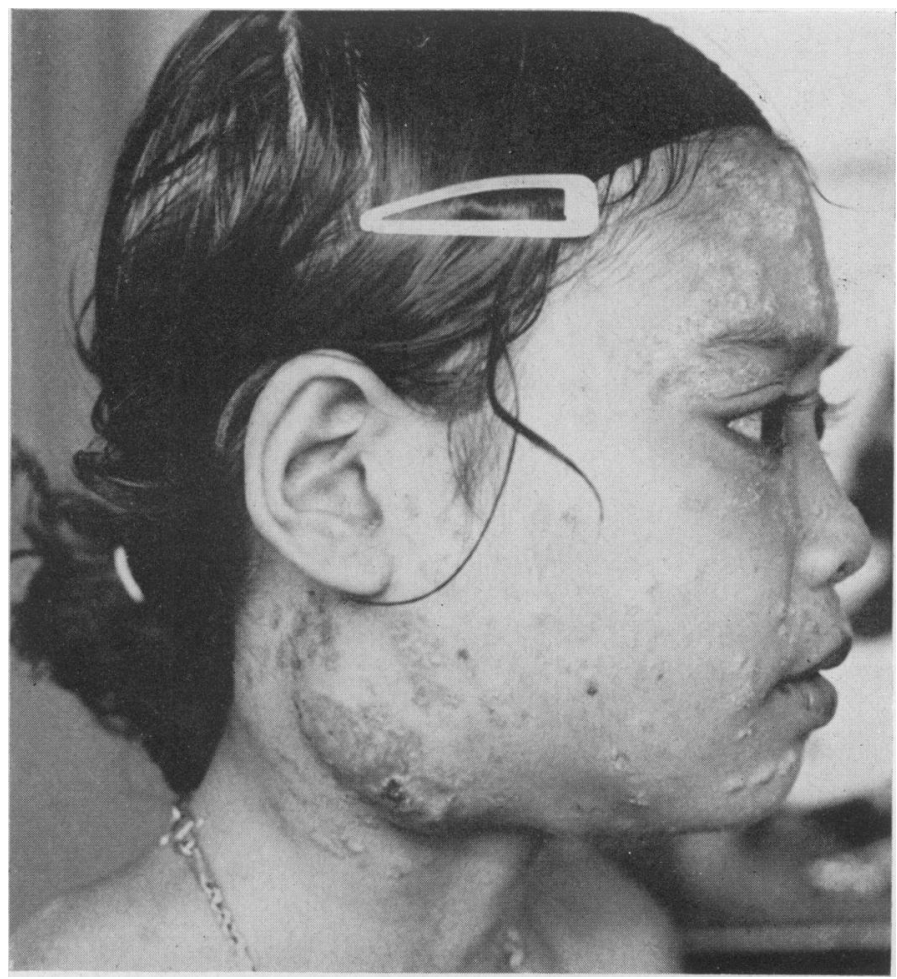

Fig. 4. Involvement of biopsy scar, 18 months after biopsy.

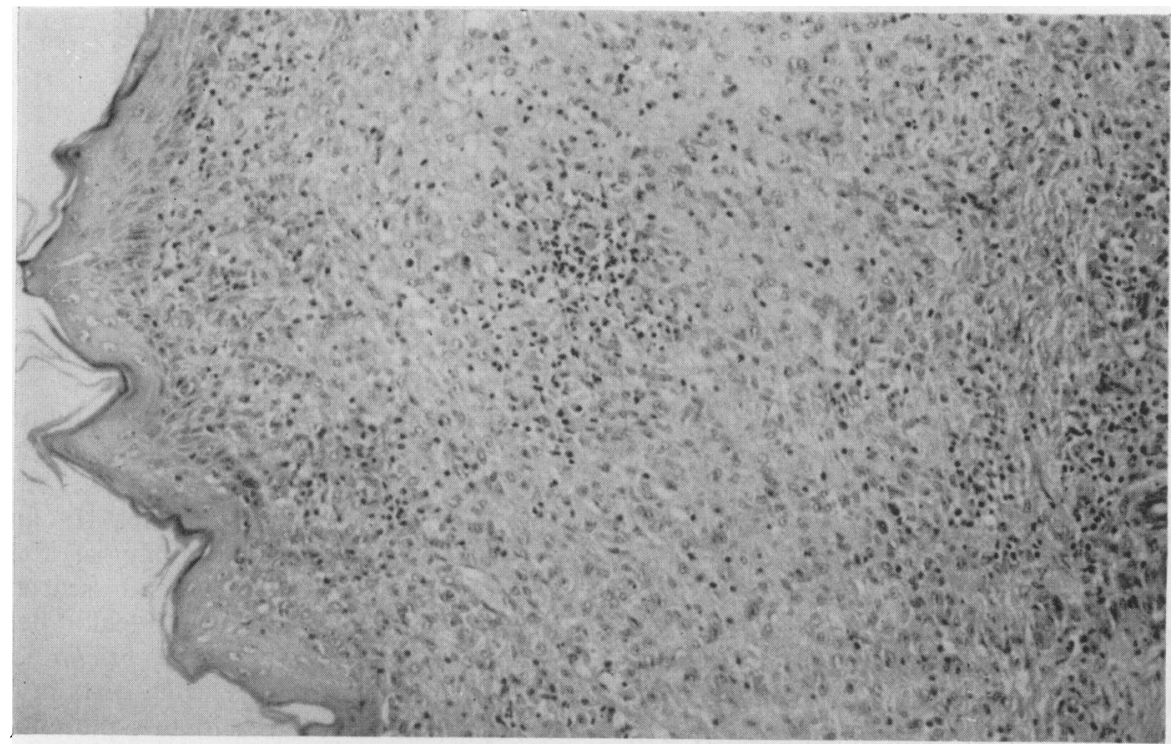

FIG. 5. Low-powered view of skin biopsy showing cellular infiltration of dermis $(\mathrm{HE}, \times 132)$. 


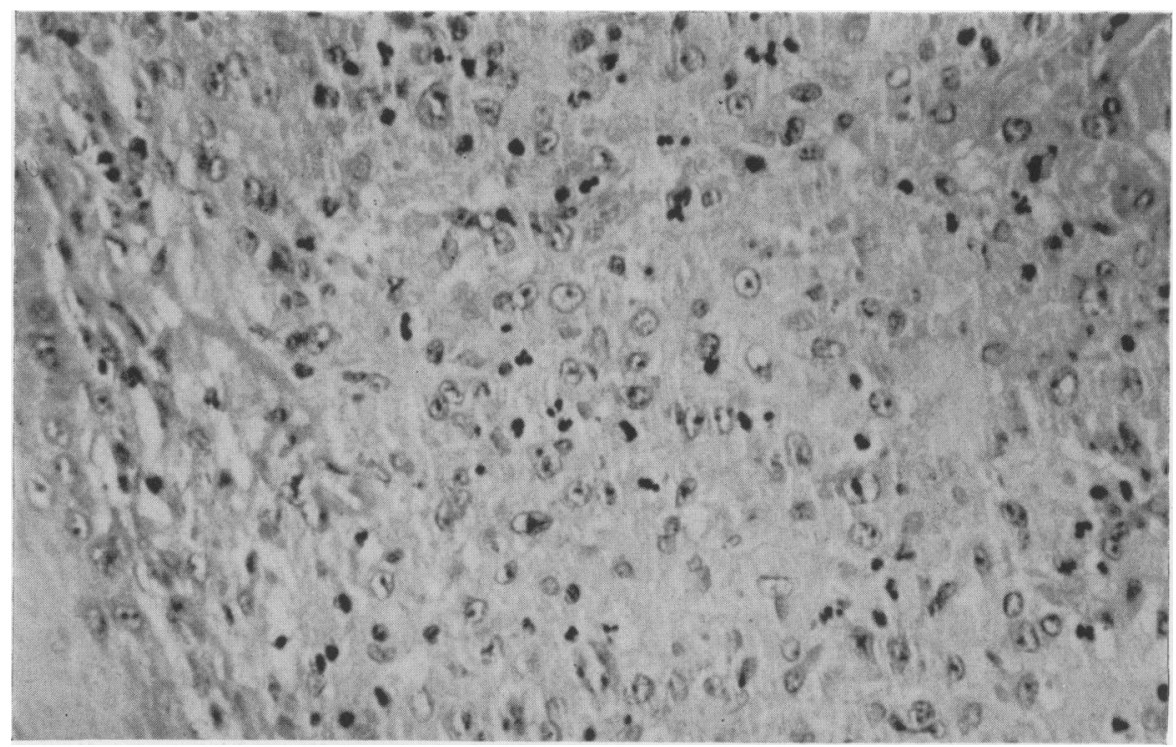

FIG. 6. High-powered view of cellular infiltration showing aggregates of histiocytes with occasional lymphocytes and plasma cells $(\mathrm{HE}, \times 330)$.

raised in other cases of SHML, and it is unlikely to be the causal agent in this case, in view of the normal Toxoplasma dye test and lack of clinical response to a 6-week trial of pyrimethamine and sulphadiazine. The benign nature of the illness is confirmed by the clinical course in this patient. The recognition of this condition is important, as demonstrated by cases which have been erroneously diagnosed and treated as malignant lymphoma, occasionally with disastrous consequences (Rosai and Dorfman, 1972).

\section{Acknowledgment}

We would like to thank Professor N. F. C. Gowing, Royal
Marsden Hospital, London, for examining the histological slides.

\section{References}

LAMPERT, F. \& LENNERT, K. (1976) Sinus histiocytosis with massive lymphadenopathy. Cancer, 37, 783.

RosaI, J. \& Dorfman, R.F. (1969) Sinus histiocytosis with massive lymphadenopathy. A newly recognised clinicopathological entity. Archives of Pathology. Chicago, 87, 63.

RosaI, J. \& Dorfman, R.F. (1972) Sinus histiocytosis with massive lymphadenopathy-a pseudo-lymphomatous benign disorder. Cancer, 30, 1174.

Thawerani, H., Sanchez, R.L., Rosai, J. \& Dorfman, R.F. (1978) The cutaneous manifestations of sinus histiocytosis with massive lymphadenopathy. Archives of Dermatology, 114, 191. 